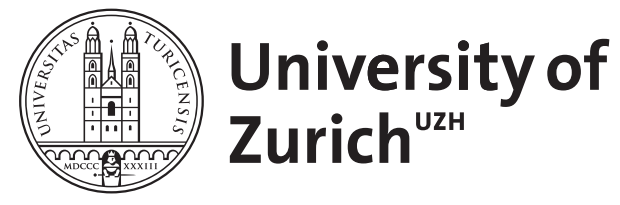

Zurich Open Repository and Archive

University of Zurich

University Library

Strickhofstrasse 39

CH-8057 Zurich

www.zora.uzh.ch

Year: 2014

2D-Raman-THz spectroscopy: A sensitive test of polarizable water models

Hamm, Peter

DOI: https://doi.org/10.1063/1.4901216

Posted at the Zurich Open Repository and Archive, University of Zurich

ZORA URL: https://doi.org/10.5167/uzh-106221

Journal Article

Accepted Version

Originally published at:

Hamm, Peter (2014). 2D-Raman-THz spectroscopy: A sensitive test of polarizable water models. Journal of Chemical Physics, 141(18):184201.

DOI: https://doi.org/10.1063/1.4901216 


\title{
2D-Raman-THz Spectroscopy: A Sensitive Test of Polarizable Water Models
}

\author{
Peter Hamm* \\ Department of Chemistry, University of Zurich, \\ Winterthurerstr. 190, CH-8057 Zürich, Switzerland \\ *peter.hamm@chem.uzh.ch
}

(Dated: October 24, 2014)

\begin{abstract}
In a recent paper, the experimental 2D-Raman- $\mathrm{THz}$ response of liquid water at ambient conditions has been presented (Proc. Natl. Acad. Sci. USA 110, 20402 (2013)). Here, all-atom molecular dynamics simulations are performed with the goal to reproduce the experimental results. To that end, the molecular response functions are calculated in a first step, and are then convoluted with the laser pulses in order to enable a direct comparison with the experimental results. The molecular dynamics simulation are performed with several different water models: TIP4P/2005, SWM4-NDP and TL4P. As polarizability is essential to describe the 2D-Raman-THz response, the TIP4P/2005 water molecules are amended with either an isotropic or a anisotropic polarizability a posteriori after the molecular dynamics simulation. In contrast, SWM4-NDP and TL4P are intrinsically polarizable, and hence the 2D-Raman- $\mathrm{THz}$ response can be calculated in a self-consistent way, using the same force field as during the molecular dynamics simulation. It is found that the 2D-Raman$\mathrm{THz}$ response depends extremely sensitively on details of the water model, and in particular on details of the description of polarizability. Despite the limited time resolution of the experiment, it could easily distinguish between various water models. Albeit not perfect, the overall best agreement with the experimental data is obtained for the TL4P water model.
\end{abstract}

\section{INTRODUCTION}

The complex hydrogen bond network, which water molecules can form, is ultimately responsible for the many thermodynamic anomalies of liquid water [1]. The intermolecular vibrations of water molecules in these hydrogen bond networks appear in a spectral range below $1000 \mathrm{~cm}^{-1}$, which has been explored extensively by both THz absorption [2-5] and Raman spectroscopy [3, 6-13]. These spectra contain three to some extent distinct features: a band centered at $\approx 600 \mathrm{~cm}^{-1}$ due to librations (i.e., hindered rotations), a band at $\approx 200 \mathrm{~cm}^{-1}$ due to hydrogen bond stretch vibrations, and a band at $\approx 60 \mathrm{~cm}^{-1}$, which is typically assigned to hydrogen bond bending modes. The librational modes are hardly visible in the Raman spectrum since the polarizability of water is almost perfectly isotropic. In contrast, the hydrogen bond bending mode is appearing only as a faint shoulder in the $\mathrm{THz}$ absorption spectrum.

To reach a deeper understanding of the ultrafast structural dynamics of liquid water, we have recently introduced a two-dimensional spectroscopy directly in this low-frequency range, coined 2D-Raman- $\mathrm{THz}$ spectroscopy $[14,15]$. The experiment is conceptually similar to 2D Raman spectroscopy, initially proposed by Tanimura and Mukamel [16], in the sense that it perturbs the system twice with two ultrashort laser pulses and as such allows one to measure three-timepoint correlation functions of the intermolecular dynamics. 2D Raman spectroscopy triggered a great deal of interest from both a theoretical [17-24] and an experimental point of view [2530]. It however turned out to be an exceptionally difficult experiment since cascaded 3rd-order processes contaminate the desired 5th-order Raman signal [31]. Together with the very weak Raman cross section of water, $2 \mathrm{D}$
Raman spectroscopy did not become feasible as of yet for water. 2D-Raman-THz spectroscopy, in contrast, can circumvent these technical problems and has in fact recently been realized experimentally for water [32].

The 2D-Raman- $\mathrm{THz}$ response is however rather complex and its interpretation is certainly not intuitive. Extracting information from these experiments requires massive support from theory as well as from molecular dynamics (MD) simulations and first steps in this direction have been taken recently $[14,15,33]$. A realistic description of the 2D-Raman- $\mathrm{THz}$ spectroscopy requires a polarizable water model for two reasons. First, trivially, polarizability is required because the spectroscopy includes a Raman interaction. A second, more subtle reason concerns the hydrogen bond stretch band at $\approx 200 \mathrm{~cm}^{-1}$ in the $\mathrm{THz}$ absorption spectrum, which is a major target of the experiment [32]. It is well established that simple point charge models of water, such as TIP4P/2005 [34] or SPC/E [35], cannot account for the intensity of that band, since that band originates from charge flows within and between water molecules upon hydrogen bonding [5, 36-39]. Adding polarizability to a water model, either in an ad hoc manner to a trajectory that has been precalculated with the help of a point-charge model [39-42], or explicitly as part of the force field [43-45], reveals the band in the $\mathrm{THz}$ absorption spectrum, albeit, often, with severely underestimated intensity.

The present paper presents a systematic study of how and to what extent various water model affect the outcome of a simulation of the 2D-Raman- $\mathrm{THz}$ response. It will be shown that the method is in particular sensitive to the level of description of polarizability of the considered water models. 


\section{BACKGROUND: MOLECULAR RESPONSE FUNCTIONS}

In 2D Raman- $\mathrm{THz}$ spectroscopy, two short laser pulses hit the sample: a non-resonant $800 \mathrm{~nm}$ pulse exciting a vibrational coherence by a Raman excitation, and a resonant half-cycle $\mathrm{THz}$ pulse exciting such a coherence directly. The coherence is then read-out by detecting the $\mathrm{THz}$ free-induction decay. Depending on which of the two laser pulses hits the sample first, it it referred to as the Raman-THz-THz or the $\mathrm{THz}-$ Raman-THz pulse sequence. The corresponding response functions are $[14$, 15]:

$$
\begin{aligned}
R^{(I)}\left(t_{2}, t_{1}\right) & \propto-\operatorname{tr}\left\{\boldsymbol{\mu}\left(t_{2}\right)\left[\boldsymbol{\mu}(0),\left[\boldsymbol{\Pi}\left(-t_{1}\right), \rho_{\text {eq }}\right]\right]\right\} \\
R^{(I I)}\left(t_{2}, t_{1}\right) & \propto-\operatorname{tr}\left\{\boldsymbol{\mu}\left(t_{2}\right)\left[\boldsymbol{\Pi}(0),\left[\boldsymbol{\mu}\left(-t_{1}\right), \rho_{\text {eq }}\right]\right]\right\}
\end{aligned}
$$

where $[. . . .$.$] is a commutator, \boldsymbol{\Pi}(\boldsymbol{t})$ and $\boldsymbol{\mu}(\boldsymbol{t})$ are polarizability and dipole operators, respectively, and $\rho_{\text {eq }}$ the equilibrium density matrix. For what follows it is convenient to define the time-point of the middle interaction as $t=0$.

The hybrid equilibrium-non-equilibrium approach introduced by Hasegawa and Tanimura [24] is used to compute these response functions from classical all-atom MD simulations. To that end, short pieces of equilibrium trajectories are calculated for the $t_{1}$-period. The momenta of the individual atoms are then perturbed at $t=0$ by plus/minus a force resulting from a $\delta$-shaped electric field pulse acting on either the dipole moment (for $R^{(I)}$ ) or the polarizibility (for $R^{(I I)}$ ). With these new initial conditions, short pieces of non-equilibrium trajectories are calculated for the $t_{2}$ period. The response functions average over very many of these events:

$$
\begin{aligned}
R^{(I)}\left(t_{2}, t_{1}\right) & \propto-\left\langle\left(\boldsymbol{\mu}_{+}\left(t_{2}\right)-\boldsymbol{\mu}_{-}\left(t_{2}\right)\right) \dot{\boldsymbol{\Pi}}\left(-t_{1}\right)\right\rangle \\
R^{(I I)}\left(t_{2}, t_{1}\right) & \propto-\left\langle\left(\boldsymbol{\mu}_{+}\left(t_{2}\right)-\boldsymbol{\mu}_{-}\left(t_{2}\right)\right) \dot{\boldsymbol{\mu}}\left(-t_{1}\right)\right\rangle
\end{aligned}
$$

where $\boldsymbol{\mu}\left(-t_{1}\right)$ and $\boldsymbol{\Pi}\left(-t_{1}\right)$ refer to the total dipole moment and polarizability, respectively, of the simulation box during the $t_{1}$-period, and $\boldsymbol{\mu}_{+}\left(t_{2}\right)$ and $\boldsymbol{\mu}_{-}\left(t_{2}\right)$ to the total dipole moments during the $t_{2}$-period in response to plus or minus the perturbing force, respectively.

For comparison, also $1 \mathrm{D}-\mathrm{THz}$ spectra are calculated from long equilibrium trajectories:

$$
I_{\mathrm{THz}}(\omega) \propto \tanh (\beta \hbar \omega / 2) \Im \int_{0}^{\infty} e^{i \omega t}\langle\boldsymbol{\mu}(t) \dot{\boldsymbol{\mu}}(0)\rangle d t
$$

where the pre-term is a quantum correction factor [46]. For 1D-Raman spectra:

$$
\begin{gathered}
I_{\text {iso }}(\omega) \propto \Im \int_{0}^{\infty} e^{i \omega t}\left\langle\Pi_{\text {iso }}(t) \dot{\Pi}_{\text {iso }}(0)\right\rangle d t \\
I_{\text {aniso }}(\omega) \propto \Im \int_{0}^{\infty} e^{i \omega t}\left\langle\operatorname{tr}\left[\boldsymbol{\Pi}_{\text {aniso }}(t) \dot{\Pi}_{\text {aniso }}(0)\right]\right\rangle d t
\end{gathered}
$$

is calculated with $\Pi_{\text {iso }}(t)=\operatorname{tr}[\boldsymbol{\Pi}(t)] / 3$ and $\boldsymbol{\Pi}_{\text {aniso }}(t)=$ $\boldsymbol{\Pi}(t)-\Pi_{\text {iso }}(t) \mathbf{1}$. The Bose-Einstein (quantum) correction factor is skipped in this case [47] since experimental Raman spectra of water are most commonly measured with the help of optical-heterodyne detected Raman-induced Kerr effect spectroscopy (OHD-RIKES) [6-8, 12, 13]. These experiments work in the time-domain, and frequency domain spectra are obtained by a Fourier transform also without that factor [7].

\section{MOLECULAR DYNAMICS DETAILS}

MD simulations were performed with the Gromacs program package [48] for the TIP4P/2005 [34] and SWM4NDP [49] water models and with a home-written code along the lines of Refs. [50-52] for the SWM4-POINT and TL4P [53] models, both of which include inducible point-dipoles which are not supported by Gromacs. A dodecahedral or a cubic box with periodic boundary conditions, respectively, was filled with 64 water molecules and simulated in the NVT ensemble at experimental density, with 2.5 fs time-step, with the Lennard Jones interactions switched to zero at longest distance allowed according to the minimum image convention. The long range electrostatic forces were either approximated by the Particle-Mesh-Ewald approximation (in the case of Gromacs) or by Ewald summation [50, 51].

To evaluate Eq. 2, consecutive pieces of 3 ps equilibrium trajectories (of which only the last 1 ps was used) were followed by in total four non-equilibrium trajectories, each 1 ps long. From that, the response functions were calculated on a grid of step size 5 fs with $0<t_{1,2}<1$ ps (only 0.25 ps are shown in Figs. 1 to 5 below, but the longer time range is needed for the convolution with the laser pulses). The strength of the perturbation at $\mathrm{t}=0$ was adjusted such that the average kinetic energy (i.e., the temperature) raised by $\approx 10 \%$. Eq. 2 was averaged over typically $\approx 2-4 \cdot 10^{7}$ such equilibrium-nonequilibrium events, amounting to a total simulation times of $\approx 150-300 \mu \mathrm{s}$.

\section{WATER MODELS}

\section{A. TIP4P/2005 amended with anisotropic polarizability}

The model considered here is different from Refs. [14, $15]$ in two regards. First, the TIP4P $/ 2005$ water model [34] is used instead of SPC/E [35], which much better reproduces the macroscopic thermodynamic properties of water. Among the simple point charge models of water, TIP4P/2005 is considered to be the most accurate one to date [54].

Second, so-called dynamical charges [36, 38] have been used to account for charge-flow effects in Refs. [14, 15]. The approach can realistically reproduce the $200 \mathrm{~cm}^{-1}$ band, but it still describes the charge flow only in an averaged sense, similar to how the dipole moment of an 
empirical point charge water model includes the average polarization in bulk water. The dynamical charges do not take into account explicitly the interactions of a given water molecule with its neighbors, and as such might be a poor representation of the spectroscopy of inhomogeneous hydrogen bond networks.

Therefore, a different approach was chosen here. One starts from the following expression to calculate the total dipole moment $\boldsymbol{\mu}$ of the simulation box $[41,55,56]$ :

$$
\mu=\sum_{i} \mu_{i}^{(0)}+\mu_{i}^{(i n d)}
$$

where the sum runs over all water molecules, $\boldsymbol{\mu}_{i}^{(0)}=$ $\sum_{k} \boldsymbol{r}_{k} q_{k}$ are the dipoles of the individual waters, and $\boldsymbol{\mu}_{\boldsymbol{i}}^{(\boldsymbol{i n d})}$ the induced dipoles:

$$
\mu_{i}^{(i n d)}=\alpha_{i} \sum_{j \neq i}\left(E_{i j}+T_{i j} \mu_{j}^{(i n d)}\right)
$$

Here, $\boldsymbol{\alpha}_{\boldsymbol{i}}$ is the polarizability tensor of the water molecule $i, \boldsymbol{E}_{\boldsymbol{i} \boldsymbol{j}}$ the electrostatic field at molecule $i$ created by all other water molecules $j$, and $\boldsymbol{T}_{\boldsymbol{i} \boldsymbol{j}}$ the dipole tensor. Eq. 6 can be solved iteratively for $\boldsymbol{\mu}_{\boldsymbol{i}}^{(\boldsymbol{i n d})}$, which is numerically tedious, in particular when the forces induced by the middle interaction in Eq. 2 need to be calculated. To simplify the calculation in analogy to Ref. [41], the dipoles of the individual waters $\boldsymbol{\mu}^{\mathbf{( 0 )}}=\sum_{k} \boldsymbol{r}_{k} q_{k}^{\prime}$ were calculated from charges $q_{k}^{\prime} \equiv q_{k} / c$ that are reduced by an empirical factor $c=1.3$ relative to that of the point charges $q_{k}$ of the TIP4P/2005 water model. This procedure effectively reduces the dipole moment of an individual water molecule to the gas phase value. Eqs. 5 and 6 is then replaced by:

$$
\boldsymbol{\mu}=\sum_{i} \boldsymbol{\mu}_{i}^{\prime(0)}+\boldsymbol{\alpha}_{i} \sum_{j \neq i} \boldsymbol{E}_{i j}
$$

where the original point charges $q_{k}$ of the water model are kept for the calculation of $\boldsymbol{E}_{\boldsymbol{i} \boldsymbol{j}}$. This approximation accounts for the second term in Eq. 6, which has been verified numerically.

The total polarizability of the simulation box is calculated by $[40-42,55,56]$ :

$$
\boldsymbol{\Pi}(t)=\sum_{i=1} \boldsymbol{\alpha}_{i}+\sum_{i \neq j} \boldsymbol{\alpha}_{i} \boldsymbol{T}_{i j} \boldsymbol{\alpha}_{j}
$$

with the same parametrization for the polarizability tensor $\boldsymbol{\alpha}_{\boldsymbol{i}}$ [57] as in Eqs. 6 and 7. In contrast to the dynamical charges used in Refs. [14, 15], dipole moment and polarizability are now described on equal footings with Eqs. 5 and 6 (which in turn are approximated by Eq. 7) on the one hand, and Eq. 8 on the other hand.

With Eqs. 7 and 8, the dipole moment $\boldsymbol{\mu}(t)$ and polarizibility tensor $\boldsymbol{\Pi}(t)$ can be calculated along the MD trajectory. Furthermore, at time $t=0$, the forces upon a $\delta$-shaped electric field pulse acting on either the dipole or the polarizability are required (Eq. 2), which can be
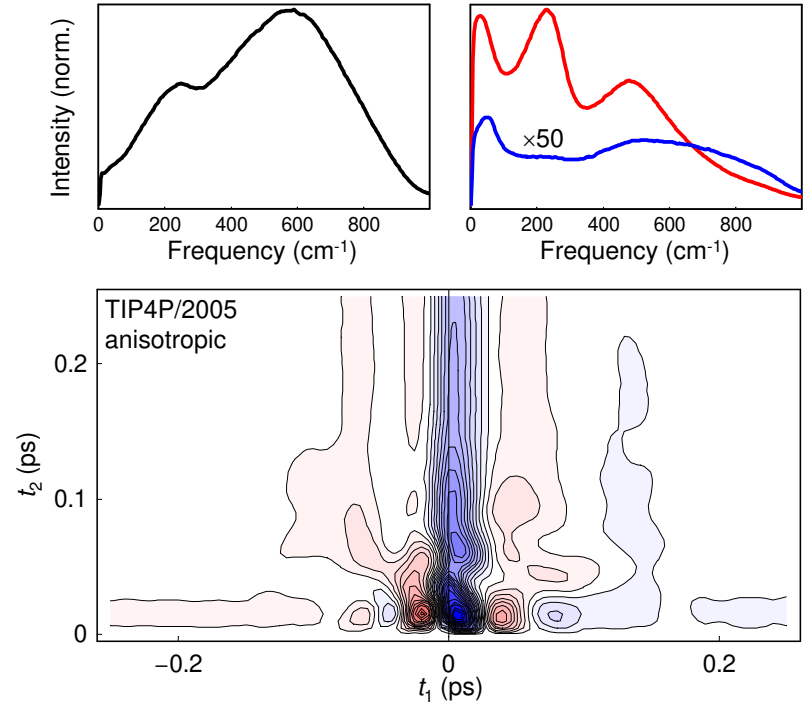

FIG. 1: TIP4P $/ 2005$ water with anisotropic polarizability [57]. Top-left panel: 1D-THz spectrum. Top-right panel: 1D Raman spectrum with the anisotropic response in red and the isotropic response in blue (the latter up-scaled by a factor 50). Bottom panel: 2D-Raman- $\mathrm{THz}$ response with the $\mathrm{THz}-$ Raman-THz pulse sequence $R^{(I I)}\left(t_{2},-t_{1}\right)$ in the left quadrant and the Raman-THz-THz pulse sequence $R^{(I)}\left(t_{2}, t_{1}\right)$ in the right quadrant. $R^{(I I)}\left(t_{2}, t_{1}\right)$ is plotted against negative times $t_{1}$ to facilitate the comparison of both response functions at $t_{1}=0$. Positive response is depicted in red, negative in blue.

calculated from analytic derivatives of Eqs. 7 or Eq. 8, respectively.

Fig. 1 shows the resulting $\mathrm{THz}$ (top-left panel) and Raman spectra (top-right panel), as well as the response functions for both the THz-Raman- $\mathrm{THz}$ (bottom panel, left quadrant) and the Raman-THz-THz pulse (bottom panel, right quadrant) sequences. The calculation of the dipole moment by Eq. 7 realistically reproduces the intensity of hydrogen-bond vibration at $200 \mathrm{~cm}^{-1}$. The $2 \mathrm{D}-$ Raman- $\mathrm{THz}$ response is qualitatively very similar to that shown in Ref. [15] (which was based on the SPC/E water model and dynamical charges to calculate the dipole moment $[36,38])$, and is practically identical to the one obtained recently by Tanimura and coworkers [33]. The latter also used the TIP4P/2005 water model and the same parametrization for the polarizability [57], but applied a full iterative calculation Eq. 6 of the dipole moment, i.e., Eq. 6 instead of Eq. 7. This agreement, in turn, evidences that the approximate treatment Eq. 7 has only a minor effect on the outcome of these simulations.

It should be noted that the parametrization of the polarizability from Ref. [57] with $a_{x x}=1.626 \AA^{3}, a_{y y}=$ $1.495 \AA^{3}$ and $a_{z z}=1.286 \AA^{3}$ (where the $x$ is the axis connecting both H's, $y$ the dipole axis, and $z$ the axis perpendicular to the water plane) strongly over-emphasizes the experimental anisotropy of the water polarizability [58]. Consequently, the intensity of the librational mode at around $600 \mathrm{~cm}^{-1}$ is by far too large in the Raman spec- 

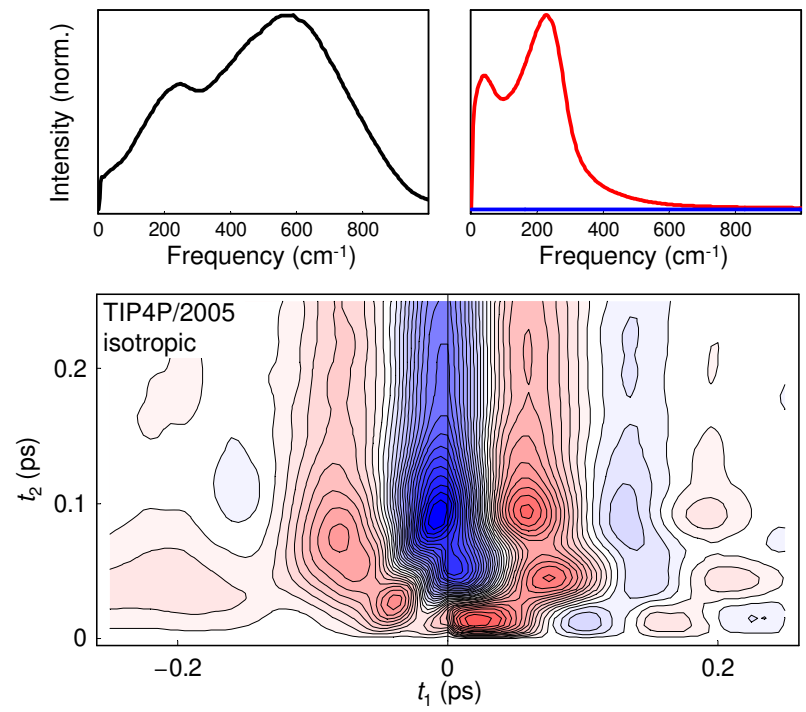

FIG. 2: TIP4P/2005 water with isotropic polarizability. Topleft panel: 1D-THz spectrum. Top-right panel: 1D Raman spectrum with the anisotropic response in red and the isotropic response in blue. Bottom panel: 2D-Raman-THz response.

tra (Fig. 1, top-right panel).

\section{B. TIP4P/2005 amended with isotropic polarizability}

In order to explore the effect of polarizability on the 2D-Raman- $\mathrm{THz}$ response, the same TIP4P/2005 water model is used in the following with the same way of calculating total dipole moment and polarizability (i.e. Eqs. 7 and 8), the only difference being that an isotropic polarizability $\boldsymbol{\alpha}_{\boldsymbol{i}}$ is used now with $a_{x x}=a_{y y}=a_{z z}=1.470 \AA^{3}$. This step will also make the connection to the intrinsically polarizable water models discussed later on, which also employ an isotropic polarizability.

The results are shown in Fig. 2. As anticipated, the $600 \mathrm{~cm}^{-1}$ band completely disappears from the anisotropic Raman spectrum (top-right panel, red line), and in fact the isotropic Raman spectrum vanishes alltogether (blue line). The latter follows from Eq. 4 and the fact that the dipole operator in Eq. 8 is traceless [8]. The $\mathrm{THz}$ spectrum (top-left panel), in contrast, is practically indistinguishable from that with anisotropic polarizability (Fig. 1).

The 2D-Raman-THz response of TIP4P with isotropic polarizability (Fig. 2, bottom panel) is substantially different from that with anisotropic polarizability (Fig. 1, bottom panel). Most prominent is a missing negative (blue) spike around $t_{1}=t_{2}=0$, which apparently originates from the librational mode that is over-emphasized in the Raman response when adding anisotropic polarizability.

\section{SWM4-NDP}

The simulations shown so far are not self-consistent, in the sense that the polarization during the MD simulation differs from that during the calculation of the response functions. One may circumvent that problem with an intrinsically polarizable water model, of which many have been developed [43-45, 49, 53, 59-69] (see Ref. [70] for a recent review that nicely summarizes existing approaches). The SWM $n$ series of water models developed by Roux, MacKerell and coworkers [49, 63, 64, 67] are among the simplest polarizable water models. They introduce polarizability via a mass-less, charged "Drude"particle attached to the oxygen atom by a harmonic spring. In the present implementation, the energy of the Drude particle is minimized iteratively at each time-step of the MD simulation, mimicking the Born-Oppenheimer approximation. These models are popular since they contain only point charges and as such can easily be implemented into standard MD codes. Here, the SWM4-NDP model [49] was chosen as it is currently the most commonly used one from the $\mathrm{SWM} n$ series of models.

The dipole moment $\boldsymbol{\mu}(t)=\sum_{k} r_{k} q_{k}$ of the simulation box can be calculated in a straight forward manner along a MD trajectory from the positions $r_{k}$ and partial charges $q_{k}$ of the various sites of the model, including those of the Drude particles. For the polarizability tensor $\boldsymbol{\Pi}(t)$, on the other hand, one makes use of $\boldsymbol{\Pi}(t)=d \boldsymbol{\mu}(t) / d \boldsymbol{E}_{\text {ext }}$, where the derivative with respect to an external electric field $\boldsymbol{E}_{\text {ext }}$ is computed numerically by finite differences. To that end, the MD trajectory is re-run with plus/minus a small external field $\boldsymbol{E}_{\text {ext }}$, re-optimizing only the Drude particles and re-calculating the total dipole moment. Finally, for the forces at $t=0$ upon a $\delta$-shaped electric field pulse acting on either the dipole moment or the polarizability, one starts with the potential energy $V=-\boldsymbol{\mu} \cdot \boldsymbol{E}_{\text {ext }}$ and $V=-\boldsymbol{E}_{\text {ext }} \cdot \boldsymbol{\Pi} \cdot \boldsymbol{E}_{\text {ext }} / 2$, respectively [33]. From that, one obtains for the transition dipole $\partial \boldsymbol{\mu} / \partial \boldsymbol{r}=d \boldsymbol{F} / d \boldsymbol{E}_{e x t}$ and for the transition polarizability $\partial \boldsymbol{\Pi} / \partial \boldsymbol{r}=d^{2} \boldsymbol{F} / d \boldsymbol{E}_{\text {ext }}^{2}$. The first and second derivatives of the forces $\boldsymbol{F}$ with respect to the external field $\boldsymbol{E}_{\text {ext }}$ are again calculated numerically by finite differences. The Gromacs suite of programs [48] supports all features needed to perform these calculations.

The results for SWM4-NDP water are shown in Fig. 3. The model severely underestimates the intensity of the $200 \mathrm{~cm}^{-1}$ band in the $\mathrm{THz}$ spectrum (top-left panel), which is present, if at all, only as a very wide shoulder extending from the librational mode towards lower frequencies (indicated by an arrow). The Raman spectra (Fig. 3, top-right panel) are dominated by the hydrogen bond bend and stretch mode, but also the librational mode gets some intensity despite the fact that the polarizability introduced by the Drude particle is isotropic. Comparison to TIP4P/2005 with isotropic polarizability (Fig. 2) as well as to SWM4-POINT and TL4P discussed below (Figs. 4 and 5) suggests that this is reflecting the fact that a induced dipole in the SWM4-NDP model is 

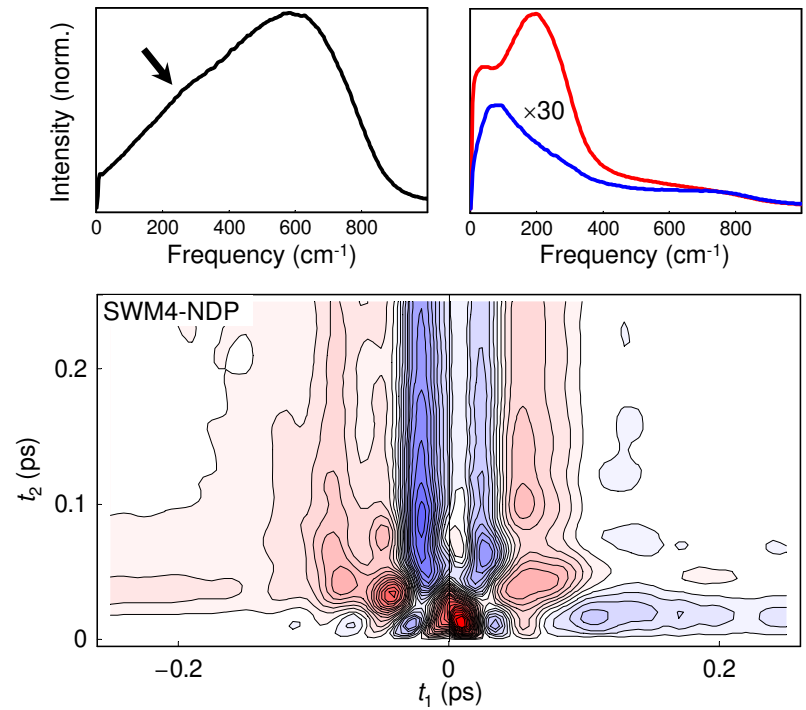

FIG. 3: SWM4-NDP water. Top-left panel: 1D-THz spectrum; the arrow indicates the hydrogen-bond stretch vibration. Top-right panel: 1D Raman spectrum with the anisotropic response in red and the isotropic response in blue (the latter up-scaled by a factor 30). Bottom panel: 2DRaman- $\mathrm{THz}$ response.

\section{SWM4-POINT}

Fig. 4 shows the results for a modified version of SWM4-NDP, where the positive and negative charges at the oxygen and the Drude particle, respectively, both giving rise to an inducible dipole, have been replaced by an inducible point-dipole with the same isotropic polarizability. All other parameters of the model are the same as for SWM4-NDP. Inducible point-dipoles are not supported by Gromacs, hence the simulation was run with a home-written MD code.

The modification has only minor effects on the structural properties of water, as evidenced by the practically indistinguishable radial distribution functions (see Fig. S1, Supplementary Material). The THz and Raman spectra (Fig. 4, top panels) of SWM4-POINT are also very similar to that of SWM4-NDP, except for the missing librational mode in the Raman spectrum, as already anticipated above. The $2 \mathrm{D}$-THZ-Raman response (Fig. 4, bottom panel), in contrast, is very different from SWM4-NDP (Fig. 3, bottom panel). Interestingly, it is much closer to that of TIP4P/2005 amended with isotropic polarizability (Fig. 2, bottom panel).
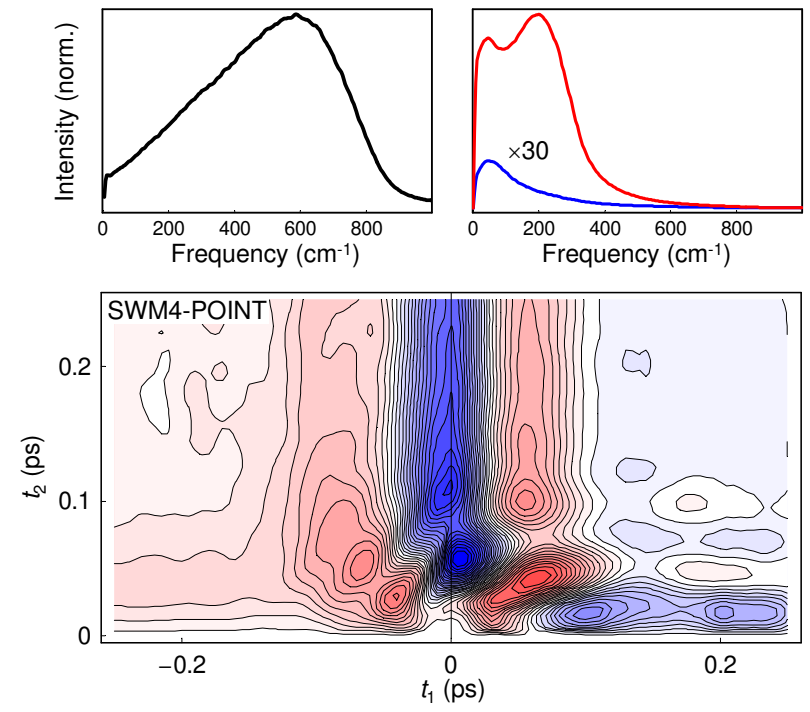

FIG. 4: SWM4-POINT water. Top-left panel: 1D-THz spectrum. Top-right panel: 1D Raman spectrum with the anisotropic response in red and the isotropic response in blue (the latter up-scaled by a factor 30). Bottom panel: 2DRaman- $\mathrm{THz}$ response.

\section{E. TL4P}

Tavan and coworkers have recently presented a series of polarizable water models, TLnP $[53,68,69]$, which feature computational simplicity together with a very accurate description of simultaneously gas-phase and liquid phase properties. Apart from a careful parametrization, the essential difference to other water models of equal complexity are Gaussian inducible dipoles [65, 71]. That solves a problem of other models such as SWM4-NDP, namely that the polarizability has to be kept smaller than the gas-phase value. A cornerstone in the parametrization of the TLnP models was to fix the dipole moment and polarizability to the corresponding experimental gas phase values (for simplicity, however, the polarizability was assumed to be isotropic). The TL4P model has been implemented into the home-written MD code, which has been verified against Ref. [53].

The results are shown in Fig. 5. Again, the model strongly underestimates the intensity of the $200 \mathrm{~cm}^{-1}$ band in the $\mathrm{THz}$ spectrum (top-left panel), which shows up only as a faint shoulder indicated by an arrow. That shoulder appears at a lower frequency than for SWM4NDP, in better agreement with experiment. From all the models, also the Raman spectra of TL4P (Fig. 5, topright panel) agree the best with experiment, in the sense that the hydrogen bond bending band at $\approx 60 \mathrm{~cm}^{-1}$ is the most intense one (in experiment, that band is actually more intense than the the hydrogen bond stretching band at $\left.\approx 200 \mathrm{~cm}^{-1}[6-8,12,13]\right)$. The 2D-Raman- $\mathrm{THz}$ response (Fig. 5, bottom panel) is yet very different from all previous models. 

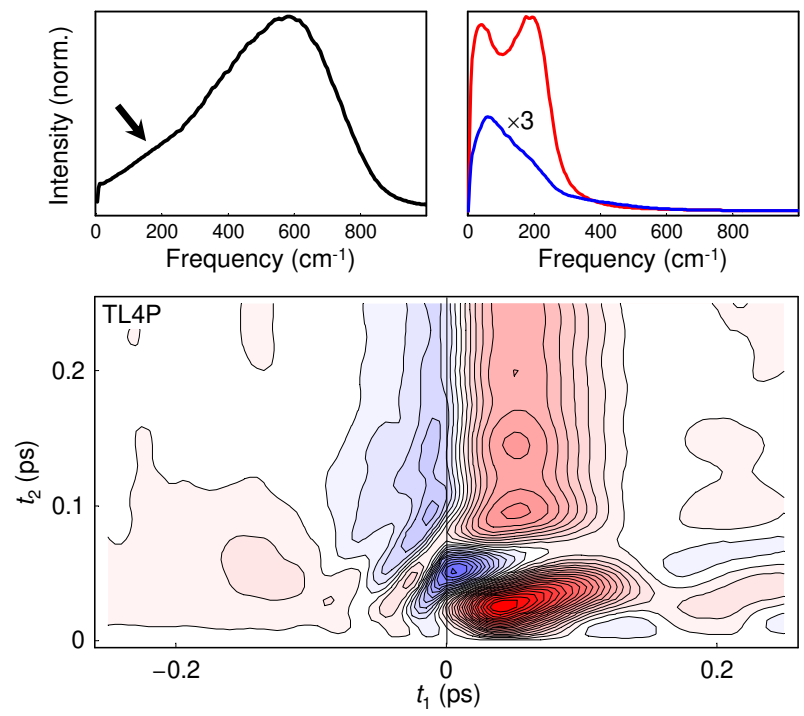

FIG. 5: TL4P water. Top-left panel: 1D-THz spectrum; the arrow indicates the hydrogen-bond stretch vibration. Topright panel: 1D Raman spectrum with the anisotropic response in red and the isotropic response in blue (the latter up-scaled by a factor 3 ). Bottom panel: 2D-Raman- $\mathrm{THz}$ response.

\section{COMPARISON TO EXPERIMENT}

Figs. 1 to 5 show the molecular response function of the various water models, which is not what is measured directly in a 2D-Raman- $\mathrm{THz}$ experiment [32]. Instead, the experimental signal is related to the convolution of the molecular response function with the laser pulses:

$$
\begin{array}{r}
P^{(3)}\left(t_{2} ; t_{1}\right)=\int_{0}^{\infty} \int_{0}^{\infty} d t^{\prime} d t^{\prime \prime} E_{T H z}\left(t_{2}-t^{\prime \prime}\right) \\
\cdot I_{\text {Raman }}\left(t_{2}+t_{1}-t^{\prime \prime}-t^{\prime}\right) R\left(t^{\prime \prime}, t^{\prime}\right),
\end{array}
$$

where $E_{T H z}$ and $I_{\text {Raman }}$ are the shapes of the $\mathrm{THz}$ and Raman pulses, respectively, and $t_{1}$ is the delay time between the peaks of these two pulses. Furthermore, the generated 3rd-order field can be described as a timederivative of the 3rd-order polarization:

$$
E^{(3)}\left(t_{2} ; t_{1}\right)=\frac{d}{d t_{2}} P^{(3)}\left(t_{2} ; t_{1}\right)
$$

which is a good approximation for the most simple case when the process is quasi-phasematched $[72,73]$. Finally, the emitted field is reshaped on the way from the sample to the detector due to dispersion and absorption of the water jet, the imaging optics and the detection crystal $[74,75]$. These effects are described by a linear transfer function $T\left(\omega_{2}\right)$ in the frequency domain, with which one obtains for the detected field:

$$
E_{\text {det }}\left(\omega_{2} ; t_{1}\right)=T\left(\omega_{2}\right) E^{(3)}\left(\omega_{2} ; t_{1}\right) .
$$

We have carefully measured the laser pulse shapes entering in Eq. 9, as well as the transfer function of Eq. 11 (see Supplementary Material of Ref. [32]).

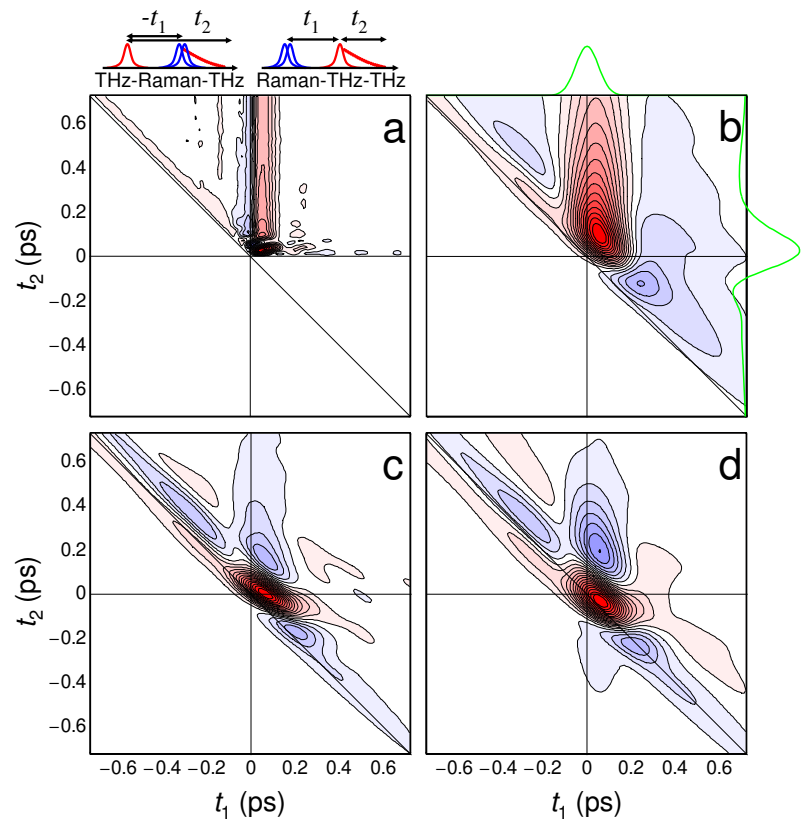

FIG. 6: From the molecular response function to the measured signal, exemplified for TL4P (see Fig. 5). (a) After transforming times for the THz-Raman- $\mathrm{THz}$ pulse sequence, (b) after convoluting with the laser pulses (Eq. 9), (c) after the $t_{2}$-derivative (Eq. 10) and (d) after applying the transfer function (Eq. 11). The pulse sequences and definitions of times are shown atop of panel (a). The green line atop of panel (b) depicts the Raman pulse $I_{\text {Raman }}$, and that on the right side the THz pulse $E_{T H z}$, both of which entering the convolution Eq. 9.

Fig. 6 illustrates what happens to the molecular response function by applying Eqs. 9 to 11, exemplified for TL4P. The first step (Fig. 6a) is related to the fact that $t_{2}$ in Eq. 9 refers to the time between the $\mathrm{THz}$ input pulse and the emitted field (see pulse sequence atop of Fig. 6a, this also reflects the experimental realization [32]), and not necessarily between the second laser pulse and the emitted field (as it does in Eq. 1). When one scans $t_{1}$ from negative to positive times, one switches from the $\mathrm{THz}-$ Raman- $\mathrm{THz}$ to the Raman- $\mathrm{THz}-\mathrm{THz}$ pulse sequence, but one has to transform time $t_{2} \leftarrow t_{1}+t_{2}$ for $R^{(I I)}$. Hence, the Raman- $\mathrm{THz}-\mathrm{THz}$ sequence appears in the upper-right quadrant with $t_{1}>0$ and $t_{2}>0$, whereas the THz-Raman-THz sequence appears in the upper triangle of the upper-left quadrant with $t_{1}<0$ and $t_{1}+t_{2}>0$.

Fig. $6 \mathrm{~b}$ shows the result of the convolution with the laser pulses (Eq. 9), for which in particular the $\mathrm{THz}$ pulse is time-limiting with a rather complicated shape (see green lines). Fig. 6c shows the result of the time derivative (Eq. 10), and Fig. 6d the final result after applying the transfer function (Eq. 11). Essentially due to the limited band-width of the detection crystal, the signal is further broadened along the $t_{2}$ direction in the last step. 

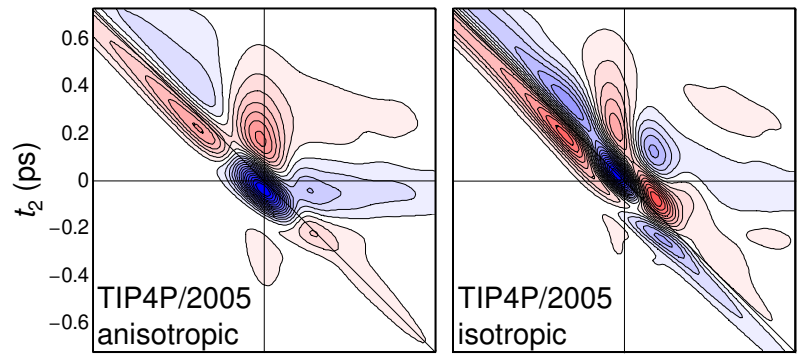

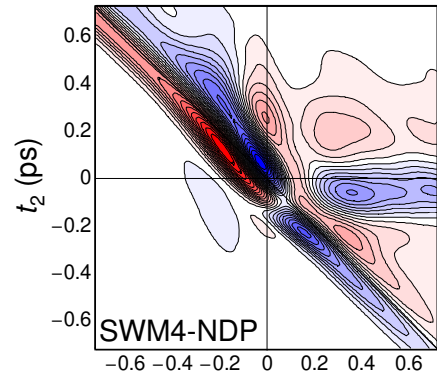

$t_{1}$ (ps)

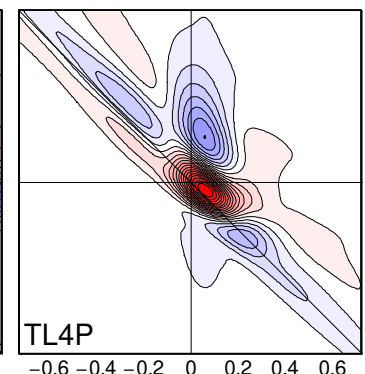

$t_{1}$ (ps)
FIG. 7: Expected outcome of a 2D-Raman-THz experiment for the various water models, calculated from Eq. 9 to 11. Top-left TIP4P/2005 with anisotropic polarizability, top-right TIP4P/2005 with isotropic polarizability, bottom left: SWM4-NDP, bottom-right: TL4P. Positive response is depicted in red, negative in blue.

Fig. 7 shows the expected outcome of an 2D-Raman$\mathrm{THz}$ experiment for the various water models. It is important to reiterate that the actual pulse shapes and the transfer function of the experiment reported in Ref. [32] have been used here, so the plots of Fig. 7 should directly be comparable to the experimental result (i.e., Fig. $2 \mathrm{~B}$ of Ref. [32]). Despite the fact that Eqs. 9 to 11, of course, smear out information significantly since the laser pulses are not infinitesimally short and the detector is not infinitesimally fast, the results for the various water models are so distinctively different that the experiment would clearly be able to distinguish them. For example, the sign of the central peak around $t_{1}=t_{2}=0$ flips when comparing TIP4P/2005 with anisotropic polarizability (Fig. 7, top-left) with TL4P (Fig. 7, bottom right). The strong dependence of the 2D-Raman- $\mathrm{THz}$ signal on the water model illustrates the extra information obtained from higher-order spectroscopy. In contrast, the 1D-THz and Raman spectra (Fig. 1 to 5, top panels) are all qualitatively the same, revealing the same spectral features with somewhat varying amplitudes.

One must conclude that none of the water models considered in this paper reveal a fully satisfactory fit with the experimental results, but overall speaking it appears that TL4P gets the closest. That conclusion refers to the sign of the major peak around $t_{1}=t_{2}=0$ (positive, red) as well as that of the wing along the $t_{2}$-axis for $t_{2}>0$ and $t_{1} \approx 0$. On the other hand, with respect to the wing along the $t_{1}$-axis (i.e., $t_{1}>0$ and $t_{2} \approx 0$ ), TIP4P $/ 2005$ amended with anisotropic polarizability seems to be bet- ter.

\section{DISCUSSION AND CONCLUSION}

The comparison of the various water models (Fig. 1 to 5 and Fig. 7) emphasizes the high sensitivity of the method. Relatively minor differences in the water model, e.g. SWM4-NDP (Fig. 3) versus SWM4-POINT (Fig. 4), can result in rather drastic changes for the 2D-Raman$\mathrm{THz}$ response. Thereby it is interesting to note that the method is more sensitive to the description of polarizability, and not necessarily to the force field per se. For example, TIP4P/2005 with isotropic polarizability (Fig. 2) and SWM4-POINT (Fig. 4) reveal quite comparable results, despite the fact that they build on considerably different force field philosophies. They both reveal comparable results, since they both describe polarizability as an inducible isotropic point-dipole situated at the oxygen site.

It furthermore appears that a realistic description of the 2D-Raman-THz response will require an anisotropic polarizability, even though the deviation from an isotropic polarizability is quite small for water so that it is typically neglected in the development of water models [49, 53]. The differences of SWM4-NDP (Fig. 3) versus SWM4-POINT (Fig. 4) emphasize this point. The polarizability of SWM4-NDP is effectively slightly anisotropic, as evidenced by the appearance of the librational mode at $\approx 600 \mathrm{~cm}^{-1}$ in the Raman spectrum, since the Drude particle moves away from the oxygen site and as such feels the anisotropic environment of a water molecule. Implementing an anisotropic polarizability into a force field in a fully consistent way would be computationally tedious, as it produced torques (and not only forces) at the sites to which a point-dipole is attached.

In any case, in light of the high sensitivity of the method, it is not too surprising that none of the considered water models reveal a truly satisfactory match with experiment for the 2D-Raman- $\mathrm{THz}$ response; they cannot even reproduce the intensity of the hydrogen-bond stretching mode at $\approx 200 \mathrm{~cm}^{-1}$ in the $1 \mathrm{D}-\mathrm{THz}$ spectrum. That band is better represented in more sophisticated polarizable models of water [43-45], presumably since those are polarizable also at the hydrogen sites and as such feel more closely the formation of a hydrogen bond, or since those are flexible models. It is however very well established that this band originates from charge flows between water molecules upon hydrogen bonding [5, 3639 , and it is quite questionable whether polarizability can describe that effect. The electric field at a polarizable site scales decays quadratically with the distance to an hydrogen bond partner, whereas charge flow effects decrease much steeper with an exponential dependence [76]. It will be interesting to explore how models along the lines of Ref. [39], which account for charge flow effects explicitly, behave in the context of 2D-Raman- 
$\mathrm{THz}$ spectroscopy.

The present study is similar in spirit to works performed by Skinner and coworkers [77], who tested various water models of equal complexity (point-charge and polarizable water models) against experimental 2D-IR spectra. Unfortunately, 2D-IR spectroscopy turned out to be rather insensitive, in the sense that all water models considered revealed reasonable agreement with experiment. The work did not result in any strong conclusions about which of the considered models better describes water.

This failure reflects the fact the 2D-IR spectroscopy is effectively a $1 \mathrm{D}$ spectroscopy with respect to the low frequency intermolecular degrees of freedom. That is, 2D-IR spectroscopy typically utilizes the $\mathrm{OH}$ or OD vibration of an HOD molecule in $\mathrm{D}_{2} \mathrm{O}$ or $\mathrm{H}_{2} \mathrm{O}$, respectively, as a local probe of its environment [78-82]. The environment is interrogated only twice, separated by one experimentally controllable time (typically called the population time). As such, 2D-IR spectroscopy measures only the two-time point correlation function of the intermolecular degrees of freedom that couple to the $\mathrm{OH}$ or the OD vibration. One can in fact describe the $2 \mathrm{D}$ IR response by the spectral density of these intermolecular degrees of freedom [83], conceptually not very different from a 1D-Raman or a 1D-THz spectrum (albeit with different selection rules). In contrast, the extension of $2 \mathrm{D}$-IR spectroscopy by one more dimension to $3 \mathrm{D}-\mathrm{IR}$ spectroscopy [84] measures a three-time point correlation function. 2D-Raman- $\mathrm{THz}$ spectroscopy is indeed conceptually similar to 3D-IR spectroscopy, but works in the low-frequency range directly without resorting to the detour via a high-frequency intramolecular vibration. The present paper illustrates how much more decisive such a three-time point correlation function can be.

Apart from a few exceptions [43-45], water models are not typically validated against experimental $\mathrm{THz}$ and/or Raman spectra. The development of polarizable water models is currently a very active field of research, because they will be a cornerstone for the next generation of MD force fields for biomolecular simulations. A very large zoo of polarizable water models exists in literature [43$45,49,53,59-70]$, with no convergence in sight as of yet towards any particular one (as it is for point charge models with TIP4P/2005 [34, 54]). Whenever polarizability is an important aspect of a water model, it appears that it should be tested against 2D-Raman- $\mathrm{THz}$ spectroscopy, given the high sensitivity of the method to in particular that property.[85]

\section{ACKNOWLEDGEMENT}

I wish to thank Yoshitaka Tanimura as well as Markus Meuwly for very valuable discussions on the topic. The experimental work, to which this paper refers [32], has been performed by Janna Savolainen and Saima Ahmed, whose contribution to the overall project is highly acknowledged. Part of the simulations have performed on the Schrödinger computer cluster of the University of Zurich. The work has been financially supported by the Swiss National Science Foundation (SNF) through the National Center of Competence and Research (NCCR) MUST.
[1] P. G. Debenedetti, J. Phys.- Condens Matter 15, R1669 (2003).

[2] J. E. Bertie and Z. Lan, Appl. Spectroscopy 50, 1047 (1996).

[3] T. Fukasawa, T. Sato, J. Watanabe, Y. Hama, W. Kunz, and R. Buchner, Phys. Rev. Lett. 95, 197802 (2005).

[4] S. Ebbinghaus, S. J. Kim, M. Heyden, X. Yu, U. Heugen, M. Gruebele, D. M. Leitner, and M. Havenith, Proc. Natl. Acad. Sci USA 104, 20749 (2007).

[5] M. Heyden, J. Sun, S. Funker, G. Mathies, H. Forbert, M. Havenith, and D. Marx, Proc. Natl. Acad. Sci. USA 107, 12068 (2010).

[6] G. E. Walrafen, M. R. Fischer, M. S. Hokmabadi, and W. H. Yang, J. Chem. Phys. 85, 6970 (1986).

[7] E. W. Castner, Jr., Y. J. Chang, and Y. C. Walrafen, J. Chem. Phys. 102, 635 (1995).

[8] C. J. Fecko, J. D. Eaves, and A. Tokmakoff, J. Chem. Phys. 117, 1139 (2002).

[9] T. Torre, P. Bartolini, and R. Righini, Nature 428, 296 (2004).

[10] N. T. Hunt, L. Kattner, R. P. Shanks, and K. Wynne, J. Am. Chem. Soc. 129, 3168 (2007).

[11] I. A. Heisler and S. R. Meech, Science 327, 857 (2010).

[12] K. Mazur, I. A. Heisler, and S. R. Meech, J. Phys. Chem.
B 115, 2563 (2011).

[13] A. Taschin, P. Bartolini, E. Eramo, R. Righini, and R. Torre, Nature Commun. 4, 2401 (2013).

[14] P. Hamm and J. Savolainen, J. Chem. Phys. 136, 094516 (2012).

[15] P. Hamm, J. Savolainen, J. Ono, and Y. Tanimura, J. Chem. Phys. 136, 236101 (2012).

[16] Y. Tanimura and S. Mukamel, J. Chem. Phys. 99, 9496 (1993).

[17] S. Palese, J. T. Buontempo, L. Schilling, W. T. Lotshaw, Y. Tanimura, S. Mukamel, and R. J. D. Miller, J. Phys. Chem. 98, 12466 (1994).

[18] S. Saito and I. Ohmine, J. Chem. Phys. 108, 240 (1998).

[19] A. Man and R. M. Stratt, Phys. Rev. Lett. 85, 1004 (2000).

[20] T. L. Jansen, J. G. Snijders, and K. Duppen, J. Chem. Phys. 113, 307 (2000).

[21] J. T. Fourkas, Adv. Chem. Phys. 117, 235 (2001).

[22] S. Saito and I. Ohmine, J. Chem. Phys. 119, 9073 (2003).

[23] R. DeVane, C. Kasprzyk, B. Space, and T. Keyes, J. Phys. Chem B 110, 3773 (2006).

[24] T. Hasegawa and Y. Tanimura, J. Chem. Phys. 125, 074512 (2006).

[25] A. Tokmakoff, M. J. Lang, D. S. Larsen, G. R. Fleming, 
V. Chernyak, and S. Mukamel, Phys. Rev. Lett. 79, 2702 (1997).

[26] D. A. Blank, L. J. Kaufman, and G. R. Fleming, J. Chem. Phys. 113, 771 (2000).

[27] L. J. Kaufman, J. Heo, L. D. Ziegler, and G. R. Fleming, Phys. Rev. Lett. 88, 207402 (2002).

[28] K. J. Kubarych, C. J. Milne, and R. J. D. Miller, Int. Rev. Phys. Chem. 22, 497 (2003).

[29] O. Golonzka, N. Demirdöven, M. Khalili, and A. Tokmakoff, J. Chem. Phys. 113, 9893 (2000).

[30] Y. L. Li, L. Huang, R. J. D. Miller, T. Hasegawa, and Y. Tanimura, J. Chem. Phys. 128, 234507 (2008).

[31] D. A. Blank, L. J. Kaufman, and G. R. Fleming, J. Chem. Phys. 111, 3105 (1999).

[32] J. Savolainen, S. Ahmed, and P. Hamm, Proc. Natl. Acad. Sci. USA 110, 20402 (2013).

[33] H. Ito, T. Hasegawa, and Y. Tanimura, J. Chem. Phys. 141 (2014).

[34] J. L. F. Abascal and C. Vega, J. Chem. Phys. 123, 234505 (2005).

[35] H. J. C. Berendsen, J. R. Grigera, and T. P. Straatsma, J. Phys. Chem. 91, 6269 (1987).

[36] A. Pasquarello and R. Resta, Phys. Rev. B 68, 174302 (2003).

[37] M. Sharma, R. Resta, and R. Car, Phys. Rev. Lett. 95 $187401(2005)$

[38] H. Torii, J. Phys. Chem. B 115, 6636 (2011).

[39] H. Torii, J. Chem. Theory Comput. 10, 1219 (2014).

[40] P. A. Madden and R. W. Impey, Chem. Phys. Lett. 123, 502 (1986).

[41] B. Guillot, J. Chem. Phys. 95, 1543 (1991).

[42] M. Souaille and J. C. Smith, Mol. Phys. 87, 1333 (1996).

[43] J. Liu, W. H. Miller, G. S. Fanourgakis, S. S. Xantheas, S. Imoto, and S. Saito, J. Chem. Phys. 135, 244503 (2009).

[44] T. Hasegawa and Y. Tanimura, J. Phys. Chem. B 115, 5545 (2011)

[45] L.-P. Wang, T. Head-Gordon, J. W. Ponder, P. Ren, J. D. Chodera, P. K. Eastman, T. J. Martinez, and V. S. Pande, J. Phys. Chem. B 107, 5933 (2013).

[46] R. Ramrez, T. López-Ciudad, P. Kumar P, and D. Marx, J. Chem. Phys. 121, 3973 (2004).

[47] M. Cho, M. Du, N. F. Scherer, G. R. Fleming, and S. Mukamel, J. Phys. Chem. 99, 2410 (1993).

[48] D. van der Spoel, E. Lindahl, B. Hess, G. Groenhof, A. E. Mark, and H. J. C. Berendsen, J. Comput. Chem. 26 1701 (2005).

[49] G. Lamoureux, E. Harder, I. V. Vorobyov, B. Roux, and A. D. MacKerrell Jr., Chem. Phys. Lett. 418, 245 (2006).

[50] M. Allen and D. Tildesley, Computer Simulation of Liquids, Oxford Science Publications (Oxford University Press, Oxford, 1987)

[51] T. M. Nymand and P. Linse, J. Chem. Phys. 112, 6152 (2000).

[52] D. Frenkel and B. Smit, Understanding Molecular Dynamics (Academic Press, San Diego, 2002).

[53] P. Tröster, K. Lorenzen, M. Schwörer, and P. Tavan, J. Phys. Chem. B 117, 9486 (2013).

[54] C. Vega and J. L. F. Abascal, Phys. Chem. Chem. Phys. 13, 19663 (2011).

[55] H. Torii, Chem. Phys. Lett. 353, 431 (2001).

[56] M. T. Sonoda, S. M. Vechi, and M. S. Skaf, Phys . Chem. Chem. Phys . 7, 1176 (2005).

[57] C. Huiszoon, Mol. Phys. 58, 865 (1986).
[58] W. F. Murphy, J. Chem. Phys. 67, 5877 (1977).

[59] P. Ahlström, A. Wallqvist, S. Engström, and B. Jönsson, Mol. Phys. 68, 563 (1989).

[60] S. W. Rick, S. J. Stuart, and B. J. Berne, J. Chem. Phys. 101, 6141 (1994).

[61] H. A. Stern, F. Rittner, B. J. Berne, and R. A. Friesner, J. Chem. Phys. 115, 2237 (2001).

[62] P. Ren and J. W. Ponder, J. Phys. Chem. B 107, 5933 (2003).

[63] G. Lamoureux and B. Roux, J. Chem. Phys. 119, 3025 (2003).

[64] G. Lamoureux, A. D. MacKerrell Jr., and B. Roux, J. Chem. Phys. 119, 5185 (2003).

[65] P. Paricaud, M. Predota, A. A. Chialvo, and P. T. Cummings, J. Chem. Phys. 122, 244511 (2005).

[66] G. S. Fanourgakis and S. S. Xantheas, J. Chem. Phys. 128, 074506 (2008)

[67] W. Yu, P. E. M. Lopes, B. Roux, and A. D. MacKerrell Jr., Chem. Phys. Lett. 138, 034508 (2013).

[68] P. Tröster, K. Lorenzen, and P. Tavan, J. Phys. Chem. B 118, 1589 (2014).

[69] P. Tröster and P. Tavan, J. Phys. Chem. Lett 5, 138 (2014).

[70] P. E. M. Lopes, B. Roux, and A. D. MacKerrell Jr., Theor. Chem. Acc. 112, 8910 (2009).

[71] D. Elking, T. Darden, and R. J. Woods, J. Comput. Chem. 28, 1261 (2007)

[72] J. T. Kindt and C. A. Schmuttenmaer, J. Chem. Phys. 110, 8589 (1999).

[73] P. Kužel, F. Kadlec, and H. Němec, J. Chem. Phys. 127, 024506 (2007).

[74] H. Němec, F. Kadlec, and P. Kužel, J. Chem. Phys. 117, 8454 (2002).

[75] J. Faure, J. van Tilborg, R. A. Kaindl, and W. P. Leemans, Optical and Quantum Electronics 36, 681 (2004)

[76] R. Kumar, J. R. Schmidt, and J. L. Skinner, J. Chem. Phys. 126, 204107 (2007)

[77] J. R. Schmidt, S. T. Roberts, J. J. Loparo, A. Tokmakoff, M. D. Fayer, and J. L. Skinner, Chem. Phys. 341, 143 (2007).

[78] J. B. Asbury, T. Steinel, K. Kwak, S. A. Corcelli, C. P. Lawrence, J. L. Skinner, and M. Fayer, J. Chem. Phys. 121, $12431(2004)$

[79] S. Yeremenko, M. S. Pshenichnikov, and D. A. Wiersma, Chem. Phys. Lett. 369, 107 (2003).

[80] M. L. Cowan, B. D. Bruner, N. Huse, J. R. Dwyer, B. Chugh, E. T. J. Nibbering, T. Elsaesser, and R. J. D Miller, Nature 434, 199 (2005).

[81] J. D. Eaves, J. J. Loparo, C. J. Fecko, S. T. Roberts, A. Tokmakoff, and P. L. Geissler, Proc. Natl. Acad. Sci. USA 102, 13019 (2005).

[82] F. Perakis and P. Hamm, J. Phys. Chem. B 115, 5289 (2011).

[83] S. Mukamel, Principles of Nonlinear Optical Spectroscopy (Oxford University Press, Oxford, 1995).

[84] S. Garrett-Roe, F. Perakis, F. Rao, and P. Hamm, J. Phys. Chem. B 115, 6976 (2011)

[85] See Supplementary Material Document No. for a comparison of the radial distribution functions of SWM-NDP and SWM4-POINT. For information on Supplementary Material, see http://www.aip.org/pubservs/epaps.html 